\title{
Proliferation of antibiotic resistance genes in coastal recirculating mariculture system
}

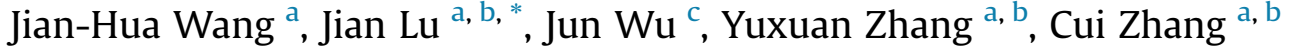 \\ ${ }^{a}$ CAS Key Laboratory of Coastal Environmental Processes and Ecological Remediation, Yantai Institute of Coastal Zone Research (YIC), Chinese Academy of \\ Sciences(CAS), Shandong Key Laboratory of Coastal Environmental Processes, YICCAS, Yantai, Shandong, 264003, PR China \\ ${ }^{\mathrm{b}}$ University of Chinese Academy of Sciences, Beijing, 100049, PR China \\ c School of Resources and Environmental Engineering, Ludong University, Yantai, Shandong, 264025, PR China
}

\section{A R T I C L E I N F O}

\section{Article history:}

Received 23 November 2018

Received in revised form

19 February 2019

Accepted 19 February 2019

Available online 25 February 2019

\section{Keywords:}

Antibiotic resistance genes

Recirculating mariculture system

Real-time quantitative PCR

Wastewater treatment

Water recycling

\begin{abstract}
A B S T R A C T
The abuse of antibiotics has caused the propagation of antibiotic resistance genes (ARGs) in aquaculture systems. Although the recirculating systems have been considered as a promising approach for preventing the coastal water pollution of antibiotics and ARG, rare information is available on the distribution and proliferation of ARGs in the recirculating mariculture system. This study firstly investigated the proliferation of ARGs in coastal recirculating mariculture systems. Ten subtypes of ARGs including tet (tetB, tetG, tetX), sul (sul1, sul2), qnr (qnrA, qnrB, qnrS), and erm (ermF, ermT) were detected. The absolute abundances of the ARGs detected in the mariculture farm were more than $1 \times 10^{4}$ copies $/ \mathrm{mL}$. The sulfonamide resistance genes (sul1 and sul2) were the most abundant ARGs with the abundance of $3.5 \times 10^{7}$ $-6.5 \times 10^{10}$ copies $/ \mathrm{mL}$. No obvious correlation existed between the antibiotics and ARGs. Some bacteria were positively correlated with two or more ARGs to indicate the occurrence of multidrug resistance. The fluidized-bed biofilter for wastewater treatment in the recirculating system was the main breeding ground for ARGs while the UV sterilization process could reduce the ARGs. The highest flux of ARGs $\left(6.5 \times 10^{21}\right.$ copies/d) indicated that the discharge of feces and residual baits was the main gateway for ARGs in the recirculating mariculture system to enter the environments.
\end{abstract}

() 2019 Elsevier Ltd. All rights reserved.

\section{Introduction}

Antibiotics are widely used in aquaculture farms because of their special role in promoting animal growth and inhibiting bacterial diseases (Xu et al., 2015). China is the largest aquaculture country in the world, accompanying with considerable abuse of antibiotics (Sapkota et al., 2008; Sharma et al., 2015). Intensive anthropogenic activities such as the aquaculture could induce the antibiotic pollution in coastal zone. Previous investigations demonstrated that aquaculture tail water was an important source of antibiotics for coastal waters (Lu et al., 2018). Antibiotics in coastal waters had caused potential ecological-health risks (Lu et al., 2018). Moreover, the use of large amounts of antibiotics in aquaculture environments induced the formation of new pollution

\footnotetext{
* Corresponding author. CAS Key Laboratory of Coastal Environmental Processes and Ecological Remediation, Yantai Institute of Coastal Zone Research (YIC), Chinese Academy of Sciences(CAS), Shandong Key Laboratory of Coastal Environmental Processes, YICCAS, Yantai, Shandong, 264003, PR China.

E-mail address: jlu@yic.ac.cn (J. Lu).
}

caused by the antibiotic resistance genes (ARGs) (Pruden et al., 2006). The emergence of ARGs makes it more difficult to control the bacterial diseases, especially those induced by the emergence of multidrug-resistant bacteria (Gao et al., 2012a; Molton et al., 2013). Therefore, the ARGs attract more attention of researchers because of the serious environmental problems induced by them.

In recent years, the aquaculture industry in China has made great progress and the mariculture mode has become more and more intensive (Yang et al., 2001). Two typical coastal mariculture modes include the conventional (without recirculation unit) and recirculating aquaculture systems (Xiong et al., 2015; Wang et al., 2018). The aquaculture wastewater in the recirculating system is circulated and reused after the systemic treatment while the wastewater in the conventional system without recirculation is discharged into coastal water directly or with simple treatment (Xiong et al., 2015; Wang et al., 2018). Recirculating aquaculture systems have been used to culture aquatic organisms in a highintensity setting since the 1960s (Bennett et al., 2018). Recirculating mariculture system has begun to become a typical intensive farming mode in recent years (van Rijn, 2013). Due to its advantages 
of less occupation, less water consumption and higher artificial control of working conditions (Piedrahita, 2003), the recirculating mariculture system is highly praised in coastal regions. Due to zero or less discharge of tailing water, the recirculating systems have been considered as an important approach for preventing the coastal water pollution of antibiotics and ARGs since antibiotics are widely applied in coastal mariculture farms to prevent diseases and promote fish growth used as feed additives (Lu et al., 2018; Wang et al., 2018).

A variety of ARGs were detected in different aquaculture systems, and considerable attention had been paid to the occurrence and abundance of ARGs (Pruden et al., 2006; Su et al., 2017; Wang et al., 2018). However, there are few reports on (1) the distribution of resistance genes in the recirculating mariculture system, (2) whether the tail water treatment technology used in the recirculating system could reduce the occurrence of ARGs, and (3) whether the ARGs produced in the recirculating system could cause a threat to the environment. To help address these knowledge gaps, here we conducted a study to investigate distribution and proliferation of ARGs in a typical coastal recirculating mariculture system. Realtime quantitative PCR (polymerase chain reaction) method was used in this study to make quantitative analysis on the typical four types of antibiotic resistance genes (tet, sul, qnr, erm) in a recirculating mariculture system. The proliferation and flow of the ARGs through all of the units in the system were also studied. This study will give some theoretical supports for assessing the environmental effects of the recirculating mariculture system and developing treatment technology of ARGs.

\section{Materials and methods}

\subsection{Sample collection}

The water and solid samples in this study were collected from a recirculating mariculture system in Yantai City, where the mariculture output accounts for $1 / 6$ of the total output in China (http:// sd.dzwww.com/sdnews/201602/t20160229_13903013.htm). The fish pond in this study is for Atlantic salmon. The recirculation ration was $90 \%$, and the UV sterilization was set for bacterial removal before entering the fish pond. The samples collected from the recirculating system were marked as $R 1, R 2, R 3, R 4, R 5, R 6$ and $R 7$. The R1, R2, R3, R4, R5 and R6 referred to the samples collected from the influent, the fish pond $\left(3000 \mathrm{~m}^{3}\right)$, the protein separator, the fluidized-bed biofilter $\left(300 \mathrm{~m}^{3}\right.$, with HRT of $1 \mathrm{~h}$ ) for wastewater treatment, the re-oxygenation pool $\left(300 \mathrm{~m}^{3}\right.$, with DO concentration exceeding $8 \mathrm{mg} / \mathrm{L}$ after re-oxygenation), and the recycled water, respectively. $\mathrm{R} 7$ was the sample of feces and residual baits collected from the fish ponds and the protein separator. Six subsamples ( $5 \mathrm{~L}$ water/sub-sample) were taken from each unit and mixed together to represent water sample of each unit. Water samples ( $30 \mathrm{~L} /$ sample) were filtered through $0.22 \mu \mathrm{m}$ micropore membrane and kept in $-80^{\circ} \mathrm{C}$ for further study.

\subsection{DNA extraction}

Water samples $(1 \mathrm{~L})$ filtered on one micropore membrane and $2.5 \mathrm{~g}$ residues were used for DNA extraction. DNA was extracted by TIANamp Soil DNA Kit (TIANGEN Biotech, Beijing, China) according to the protocol of the manufacturer. The concentrations and purity of DNA were monitored on 1\% agarose gels and NanoDrop Lite (Thermo, USA). DNA was diluted to $1 \mathrm{ng} / \mu \mathrm{L}$ using TE buffer for further study.

\subsection{PCR assays for detection of ARGs}

The primers used by this study selected from previous publications (Xu et al., 2017a; Zhu et al., 2017) were listed in Table S1. PCR assays targeting at the $16 \mathrm{~S}$ rRNA, integrase gene I1 (intI1) gene and 30 ARGs including 3 sulfonamide (sul1, sul2, sul3), 18 tetracycline (tet $\mathrm{A}$, tet $\mathrm{B}$, tetC, tet $\mathrm{D}$, tet $\mathrm{E}$, tet $\mathrm{H}$, tet $\mathrm{G}$, tet $\mathrm{J}$, tet $\mathrm{K}$, tet $\mathrm{L}$, tet $\mathrm{M}$, tet $\mathrm{O}$, tet $\mathrm{Q}$ tetR, tetS, tet $\mathrm{T}$, tet $\mathrm{W}$, tet $\mathrm{X}), 4$ quinolone ( $q n r \mathrm{~A}, q n r \mathrm{~B}, q n r \mathrm{D}, q n r \mathrm{~S})$ and 5 macrolide (ermA, ermB, erm $\mathrm{C}$, erm $\mathrm{F}$, erm $\mathrm{T}$ ) resistance genes were conducted using Eastwin Thermal Cycler (Eastwin, China). Each $20 \mu \mathrm{L}$ PCR reaction mixture buffer contained dNTP $(400 \mu \mathrm{M})$, forward and reverse primers $(100 \mu \mathrm{M}), 1 \mathrm{U}$ Taq polymerase, and $1 \mu \mathrm{L}$ template DNA. The PCR were performed using the following procedure: 2 min at $95^{\circ} \mathrm{C}$, followed by 30 cycles $\left(95^{\circ} \mathrm{C}\right.$ for $30 \mathrm{~s}, 55^{\circ} \mathrm{C}$ for $30 \mathrm{~s}$, and $72{ }^{\circ} \mathrm{C}$ for $30 \mathrm{~s}$ ), an extension at $72{ }^{\circ} \mathrm{C}$ for $10 \mathrm{~min}$, and then held at $4{ }^{\circ} \mathrm{C}$. Both positive and negative controls were included in each PCR run. The PCR products were checked on $2 \%$ agarose gel and purified with Qiagen Gel Extraction Kit (Qiagen, Germany).

\subsection{Quantitative real-time PCR ( $q P C R)$}

Quantitative real-time PCR (qPCR) was used to determine the abundance of ARGs, intI1 gene and 16S rDNA in the mariculture farm. Calibration standard curves for positive controls and quantification were made by the plasmids DNA (cloned ARGs) extracted from $E$. coli DH5 $\alpha$ as described previously (Pei et al., 2006; Luo et al., 2010). The qPCR amplification reactions were performed in 384well plates with a final volume of $25 \mu \mathrm{L}$ of reaction mixture including SYBR Premix Ex Taq II (Takara, Dalian, China), forward and reverse primers $(0.4 \mu \mathrm{M})$, and $2 \mu \mathrm{L}$ DNA template. All qPCR amplification and quantification processes were conducted using Bio-Rad IQ5 real-time PCR system (Bio-Rad, CA, USA) with the following protocol: $30 \mathrm{~s}$ at $95^{\circ} \mathrm{C}$, followed by 40 cycles at $95^{\circ} \mathrm{C}$ for $5 \mathrm{~s}, 30 \mathrm{~s}$ at the annealing temperature, a $72{ }^{\circ} \mathrm{C}$ extension for $40 \mathrm{~s}$, and then a final melt curve stage with temperature ranging from 60 to $95^{\circ} \mathrm{C}$. Each reaction was run in triplicate. The standard curve for each qPCR assay consisted of 10 -fold dilution series from $10^{7}$ to 1 copies $/ \mu \mathrm{L}$. The amplification efficiencies ranged from $91.5 \%$ to $109.3 \%$ and the $\mathrm{R}^{2}$ values were more than 0.99 for all standard curves.

\subsection{S rRNA gene high-throughput sequencing}

The extracted DNA samples were sent directly to Novegene (Beijing, China) for Illumina MiSeq sequencing. The V4-V5 hypervariable regions of the bacteria 16S rRNA gene were selected for amplification with primers 515F (5'- GTGCCAGCMGCCGCGG-3') and 907R ( $5^{\prime}$-CCGTCAATTCMTTTRAGTTT- $\left.3^{\prime}\right)$. The sequences were generated on an Illumina HiSeq2500 platform and quality filtering on the raw tags was performed by QIIME (V1.7.0). Sequence analysis was performed by Uparse software (Uparse v7.0.1001). Sequences with $\geq 97 \%$ similarity were assigned to the same OTUs. Representative sequence for each OTU was screened for further annotation. For each representative sequence, the GreenGene Database was used based on RDP classifier (Version 2.2) algorithm to annotate taxonomic information. The sequences derived from highthroughput sequencing were deposited in the National Center for Biotechnology Information (NCBI) database under accession number SRP128966.

\subsection{Antibiotics analysis}

The concentrations of 17 antibiotics belonging to the tetracycline, sulfonamide, quinolone and macrolide families were analyzed by high-performance liquid chromatography-tandem 
mass spectrometry (HPLC-MS/MS) according to the methods described by Huang et al. (2013) and Lu et al. (2018). The detailed information on sample extraction procedure was provided by previous investigations (Huang et al., 2013; Lu et al., 2018). The water samples were filtered using $0.45 \mu \mathrm{m}$ membrane filters (Pall Life Sciences, Ann Arbor, MI, USA) and spiked by internal standards with concentration of $100 \mu \mathrm{g} / \mathrm{L}$. Solid phase extraction was used to prepare sample. Oasis hydrophilic-lipophilic balance (HLB) cartridges $(6 \mathrm{~cm} / 500 \mathrm{mg})$ purchased from Waters Corporation (Milford, MA, USA) were used for extracting target antibiotics from the mariculture water samples. The final extracts were analyzed using a Shimdzu UPLC (Kyoto, Japan) equipped with a AB Sciex API 3000 tandem mass (MS/MS) detector (Redwood City, CA, USA). Instrument and analysis procedures used by this study were same with Huang et al. (2013). Concentrations of target antibiotics were determined by the average values of three replicate measurements. The recoveries of all the target antibiotics in the quality-control water samples ranged from $75.0 \%$ to $126.0 \%$ on average. The limit of detection (LOD) and limit of quantification (LOQ) for the target antibiotics were in the range of $0.9-3.57 \mathrm{ng} \mathrm{L}^{-1}$ and $4.9-11.9 \mathrm{ng} \mathrm{L}^{-1}$, respectively.

\subsection{Statistical analysis}

Pearson correlation analysis was used to investigate the potential relationship between ARGs and the corresponding antibiotics by SPSS 19.0 (IBM, New York, USA). The redundancy analysis (RDA) was performed to show the relationship between ARGs or samples with environmental variables by ggplot 2 package in $\mathrm{R}$ software. The network analysis was performed by Cytoscape 3.6.1 to investigate the relationship among bacteria, antibiotics, and ARGs.

\section{Results and discussion}

\subsection{Prevalence of ARGs in the coastal recirculating mariculture farm}

Among the 30 ARGs considered, 10 ARGs subtypes including tet (tetB, tetG, tetX), sul (sul1, sul2), qnr (qnrA, qnrB, qnrS), and erm (ermF, erm $\mathrm{T}$ ) were chosen for further quantitative analysis due to their high detection frequencies (100\%) in all mariculture units. The abundances of various ARGs, intI1 gene and 16S-rRNA were determined to characterize general trends in their proliferation and removal through pond and different treatment units. The relative abundance heatmaps of these genes were illustrated in Fig. 1. The

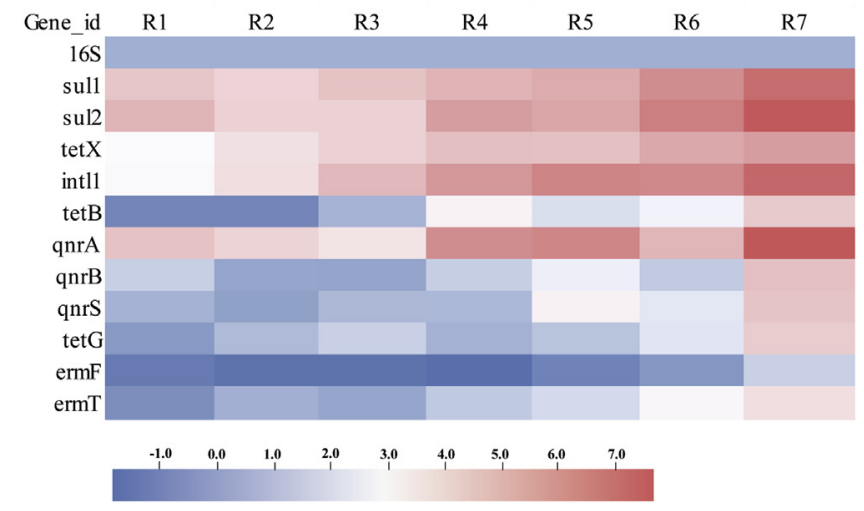

Fig. 1. Heat map of the relative abundance of the ARGs, intI1 and 16S-rRNA in each sample. The samples R1, R2, R3, R4, R5, R6 and R7 refer to the influent, water from fish pond, water from protein separator, water from fluidized-bed biofilter for wastewater treatment, water from re-oxygenation pool, the recycled water, and feces and residual baits collected from the fish ponds and the protein separator, respectively. heatmaps indicated that sul, sul2, tetX, qnrA and intI1 were generally detected with higher abundances than other ARGs. These ARGs were commonly found in aquaculture environment. The ARGs of sul (sul1, sul2, sul3, sul/folP), tet (tetA, tetB, tetC, tetD), and qnr (qnrA, $q n r \mathrm{~B}, q n r \mathrm{~S}, q n r \mathrm{D})$ were frequently detected at the coastal mariculture sites (Gao et al., 2018). Jang et al. (2018) found tet (tetA, tetB, tet $\mathrm{D}$, tet $\mathrm{E}$, tet $\mathrm{G}$, tet $\mathrm{H}$, tet $\mathrm{M}$, tet $\mathrm{Q}$, tet $\mathrm{X}$, tetZ, tetBP), sul (sul1, sul2), qnr ( $q n r \mathrm{~S}, q n r \mathrm{D})$, and erm (erm $\mathrm{C}$ ) in the effluents of coastal mariculture in South Korea.

\subsection{The absolute abundance of ARGs and intI1 in recirculating mariculture farm}

The absolute abundances of four types of ARGs (sul, tet, qnr, erm) and intI 1 were illustrated in Table 1 . The absolute abundances of all target ARGs detected in the mariculture farm were more than $1 \times 10^{4}$ copies/mL. Sulfonamide resistance genes (sul1 and sul2) were the most abundant in the recirculating mariculture farm with the total abundance of $3.5 \times 10^{7}-6.5 \times 10^{10}$ copies $/ \mathrm{mL}$ in all the samples. The sul genes were also detected at high concentration of $(1.7 \pm 0.2) \times 10^{11}$ copies/g in the sediment of Haihe River which was the receiving river of agriculture wastewater (Luo et al., 2010). The quinolone and tetracycline resistance genes were also abundant in the aquaculture farm. The total absolute abundances of $q n r$ genes ( $q n r \mathrm{~A}$, qnrB and qnrS) were $9.9 \times 10^{6}-6.4 \times 10^{10} \mathrm{copies} / \mathrm{mL}$ while those of the tet genes (tet $\mathrm{B}$, tet $\mathrm{G}$ and tet $\mathrm{X}$ ) were $5.0 \times 10^{5}-1.6 \times 10^{8}$ copies/mL for every unit in the farm. The ARGs tet and qnr were also found at high abundance levels in wastewater treatment plants with the abundances in the range of $(7.3 \pm 9.6) \times 10^{3}$ $(1.3 \pm 1.6) \times 10^{10}$ copies/mL (Mao et al., 2015). The macrolide resistance genes showed the lowest absolute abundances among the four kinds of ARGs. The total absolute abundances of erm genes (ermF and ermT) were $6.0 \times 10^{3}-1.1 \times 10^{6}$ copies/mL (Table 1) through every unit in the farm. The erm genes were detected at high abundances of $1.0 \times 10^{8}-1.0 \times 10^{14}$ copies $/ \mathrm{mL}$ in swine wastewater treatment system (Sui et al., 2017).

The intI1 played important roles in the emergence and horizontal transfer of ARGs among bacteria, and it was the main transmissible vector commonly found in different environments (Xu et al., 2017a). The intI1 gene was very abundant in the mariculture farm with the resolute abundances ranging from $4.3 \times 10^{5}$ to $1.3 \times 10^{10}$ copies $/ \mathrm{mL}$ (Table 1 ) in units of the farm. The absolute abundance of intI 1 in Xiangjiang River was up to $9.1 \times 10^{6}$ copies/ $\mathrm{mL}$ (Xu et al., 2017a). The relative high abundance of intI 1 suggested the potential high mobility of the ARGs in the recirculating mariculture farm.

\subsection{Influence of antibiotics and water quality on the ARGs in recirculating mariculture farm}

Fig. 2a showed the concentrations of the four kinds of antibiotics and abundances of their corresponding ARGs in the recirculating mariculture system. The concentrations of sulfonamides were very low, ranging from ND (not detected) to $0.04 \mu \mathrm{g} / \mathrm{L}$. The antibiotics of quinolone $(5.50-8.36 \mu \mathrm{g} / \mathrm{L})$ and tetracycline $(5.73-5.96 \mu \mathrm{g} / \mathrm{L})$ were at high concentration levels. Additionally, the concentrations of macrolides ranged from 0.08 to $0.09 \mu \mathrm{g} / \mathrm{L}$. The concentrations of the antibiotics including quinolone, tetracycline, and macrolide in the recurculating mariculture system were relatively stable, indicating these antibiotics were resistant in the various water treatment processes. The Pearson correlation analysis showed that there were significant correlation between these antibiotics $\left(r^{2} \geq 0.999\right)$ (Table 2), suggesting that wide application of antibiotics in aquaculture systems might be the main pollution source for these contaminants. 
Table 1

Abundance of ARGs of every operation unit in the recirculating mariculture farm (copies $/ \mathrm{mL}$ ).

\begin{tabular}{|c|c|c|c|c|c|c|c|}
\hline Gene_id & $\mathrm{R} 1$ & $\mathrm{R} 2$ & R3 & $\mathrm{R} 4$ & R5 & R6 & R7 \\
\hline $16 S$ & $1.959 \times 10^{4}$ & $4.063 \times 10^{4}$ & $7.655 \times 10^{4}$ & $8.864 \times 10^{4}$ & $1.929 \times 10^{4}$ & $4.454 \times 10^{3}$ & $5.340 \times 10^{3}$ \\
\hline sul1 & $2.001 \times 10^{7}$ & $1.602 \times 10^{7}$ & $1.065 \times 10^{8}$ & $3.555 \times 10^{8}$ & $1.586 \times 10^{8}$ & $3.884 \times 10^{8}$ & $7.194 \times 10^{9}$ \\
\hline sul2 & $6.999 \times 10^{7}$ & $1.865 \times 10^{7}$ & $3.709 \times 10^{7}$ & $2.033 \times 10^{9}$ & $2.306 \times 10^{8}$ & $1.388 \times 10^{9}$ & $5.794 \times 10^{10}$ \\
\hline tet $B$ & $2.936 \times 10^{3}$ & $5.916 \times 10^{3}$ & $9.187 \times 10^{4}$ & $3.669 \times 10^{6}$ & $1.509 \times 10^{5}$ & $8.062 \times 10^{4}$ & $4.142 \times 10^{6}$ \\
\hline tetG & $7.404 \times 10^{3}$ & $6.920 \times 10^{4}$ & $3.321 \times 10^{5}$ & $9.914 \times 10^{4}$ & $4.574 \times 10^{4}$ & $4.481 \times 10^{4}$ & $3.761 \times 10^{6}$ \\
\hline tetX & $4.936 \times 10^{5}$ & $6.341 \times 10^{6}$ & $3.623 \times 10^{7}$ & $1.537 \times 10^{8}$ & $2.958 \times 10^{7}$ & $4.734 \times 10^{7}$ & $1.185 \times 10^{8}$ \\
\hline$q n r A$ & $2.521 \times 10^{7}$ & $1.674 \times 10^{7}$ & $9.767 \times 10^{6}$ & $7.236 \times 10^{9}$ & $3.338 \times 10^{9}$ & $1.474 \times 10^{7}$ & $6.422 \times 10^{10}$ \\
\hline$q n r B$ & $7.803 \times 10^{4}$ & $2.536 \times 10^{4}$ & $4.484 \times 10^{4}$ & $3.470 \times 10^{5}$ & $2.909 \times 10^{5}$ & $1.468 \times 10^{4}$ & $8.299 \times 10^{6}$ \\
\hline qnrS & $2.259 \times 10^{4}$ & $2.062 \times 10^{4}$ & $1.197 \times 10^{5}$ & $1.355 \times 10^{5}$ & $8.961 \times 10^{5}$ & $4.922 \times 10^{4}$ & $6.176 \times 10^{6}$ \\
\hline ermF & $1.668 \times 10^{3}$ & $2.386 \times 10^{3}$ & $4.434 \times 10^{3}$ & $3.817 \times 10^{3}$ & $2.546 \times 10^{3}$ & $1.461 \times 10^{3}$ & $2.283 \times 10^{4}$ \\
\hline ermT & $4.322 \times 10^{3}$ & $4.178 \times 10^{4}$ & $4.756 \times 10^{4}$ & $2.597 \times 10^{5}$ & $1.215 \times 10^{5}$ & $1.453 \times 10^{5}$ & $1.048 \times 10^{6}$ \\
\hline intI1 & $4.323 \times 10^{5}$ & $7.600 \times 10^{6}$ & $2.161 \times 10^{8}$ & $3.046 \times 10^{9}$ & $3.758 \times 10^{9}$ & $5.784 \times 10^{8}$ & $1.301 \times 10^{10}$ \\
\hline
\end{tabular}

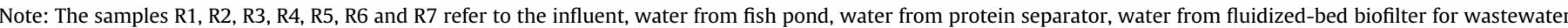

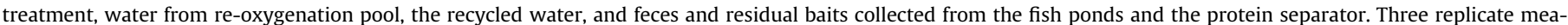
surements were made, and the average values were listed here.

No obvious correlation existed between the antibiotics and their corresponding resistance genes. The results indicated that the emergence of resistance genes were deduced by multiple factors, rather than solely caused by the abuse of antibiotics in this system. Many studies indicated that ARGs were correlated with many factors (An et al., 2018; Jiao et al., 2017; Xu et al., 2017; Yang et al., 2017). According to previous investigation, correlation between tet genes and tetracyclines were considerably weak in municipal wastewater treatment plant (Gao et al., 2012b). However, other investigations demonstrated that absolute tet genes copies (sum of tet $\mathrm{M}$, tetO, tet $\mathrm{Q}$, tet $\mathrm{W}$ ) were strongly correlated with the concentrations of tetracyclines residues in soil (Wu et al., 2010). There were not obvious correlation between the antibiotics and the intI 1 gene which could facilitate ARGs prevalence and horizontal transfer. However, the high correlation between the intI 1 and ARGs was observed $\left(r^{2}>0.923\right)$, confirming the high mobility of these detected ARGs in the recirculating mariculture systems.

The RDA analysis (Fig. 2b) was performed to identify the correlation between ARGs and water environment variables such as phosphate (P), total phosphorus (TP), ammonium $\left(\mathrm{NH}_{3}^{+}\right)$, nitrate $\left(\mathrm{NO}_{3}^{-}\right)$, nitrite $\left(\mathrm{NO}_{2}^{-}\right)$, and total carbon (TOC). The RDA analysis showed that the ARGs abundances were positively influenced by the nutrient levels in water, suggesting that the increase in the water nutrient levels could enhance the accumulation of the ARGs. Water source could be an important medium disseminating ARGs to the aquaculture environment (Su et al., 2017). The Pearson correlation analysis also showed that there were significant correlations between these ARGs $\left(r^{2} \geq 0.923\right)$, confirming that the proliferation of ARGs in aquaculture systems could occur under similar conditions.

\subsection{Relationship between bacterial community and ARGs in recirculating mariculture farm}

Network analysis of the correlation between bacteria and ARGs showed that Polaribacter, Pseudofulvibacter, Colwellia and NS10_marine_group were hot genera relating with more than 8 other genera in this system (Fig. 3). The bacteria Marinomonas (relating to sul1, sul2, tetX, tetB, qnrA, qnrB and ermT), Celeribacter (relating to sul1, tetX, tetB, intI1, qnrS and ermT), Desulfobacterium (relating to sul1, sul2, tetX, tetG and ermT), Neptuniibacter (relating to sul1, sul2, tetX and tetB), Marinicella (relating to sul1, intI1, qnrS, tetG and ermT), Colwellia (relating to qnrS and tetG), and Halobacteriovorax (relating to qnrA and qnrB) were correlated positively with two or more ARGs, indicating that these genera might be multidrug-resistant bacteria. There were positive relationship between tet genes (tet $\mathrm{X}$, tet $\mathrm{B}$ and tet $\mathrm{G}$ ) and genera Colwellia,
Marinomonas, Spirochaeta_2, Celeribacter, Paraglaciecola, Leucothrix, Desulfobacterium, Neptuniibacter and Marinicella. Results indicated that these bacteria might carry with the tet genes. The sul genes (sul1 and sul2) were correlated with bacteria Marinomonas, Celeribacter, Desulfobacterium, Neptuniibacter, and Marinicella. The $q n r$ genes ( $q n r \mathrm{~A}, q n r \mathrm{~B}$ and $q n r S$ ) were correlated with bacteria Donghicola, Colwellia, Marinomonas, Oleispira, Halobacteriovorax, unidentified_Rhodobacteraceae, Neptunomonas, and Marinicella. The ermT were correlated with bacteria Marinomonas, Celeribacter, Desulfobacterium, and Marinicella. Similarly, it was hypothesized that these bacteria carried the ARGs which were positively associated with them. The gene of intI 1 was positively correlated with bacteria Celeribacter and Marinicella. Most of ARGs detected in a peri-urban river were significantly correlated with Chloroflexi, Firmicutes, Gennatimonadetes, Plantcyomycetes and Verrucomicrobia (Zheng et al., 2018). Correlation analysis and host analysis showed that the changes in the abundances of several genera like Prevotella and Treponema were positively correlated with the antibiotic resistome alteration in livestock breeding wastewater and its receiving river (Jia et al., 2017). Another study indicated that bacteria played a dominant role in the accumulation of ARGs in manure-treated soil (Peng et al., 2016). It was also found that bacterial community shifts played an important role in shaping the antibiotic resistome in soil (Chen et al., 2016).

\subsection{Proliferation of ARGs through various treatment units in the recirculating mariculture farm}

The two samples with the highest abundances of ARGs were R7 (feces and baits residues) and R4 (fluidized-bed biofilter water), and then followed by the samples R6 (recycled water) and R5 (reoxygenation pool water) (Fig. 4a). The results showed that the feces and residual baits were an important reservoir for ARGs in the recirculating mariculture system. The fluidized-bed biofilter became the most important breeding ground for ARGs. The ARGs abundance in R6 sample sharply decreased after the processes of biochemical treatment and biological aeration, indicating that UV sterilization could reduce the ARGs abundance. The abundances of ARGs in R1, R2 and R3 were lower than those in other samples, indicating that the source water, fish pond and the protein separator were not the main breeding grounds for ARGs. However, the abundances of tet and sul genes increased obviously in R3 versus $\mathrm{R} 2$, indicating that the protein separator process could lead to the increase of these two types of genes. Previous studies showed that the current sewage treatment process could remove ARGs and the removal efficiency was affected by many factors such as treatment method (Zhang et al., 2016; Xu et al., 2017b; Wen et al., 2016; Chen 

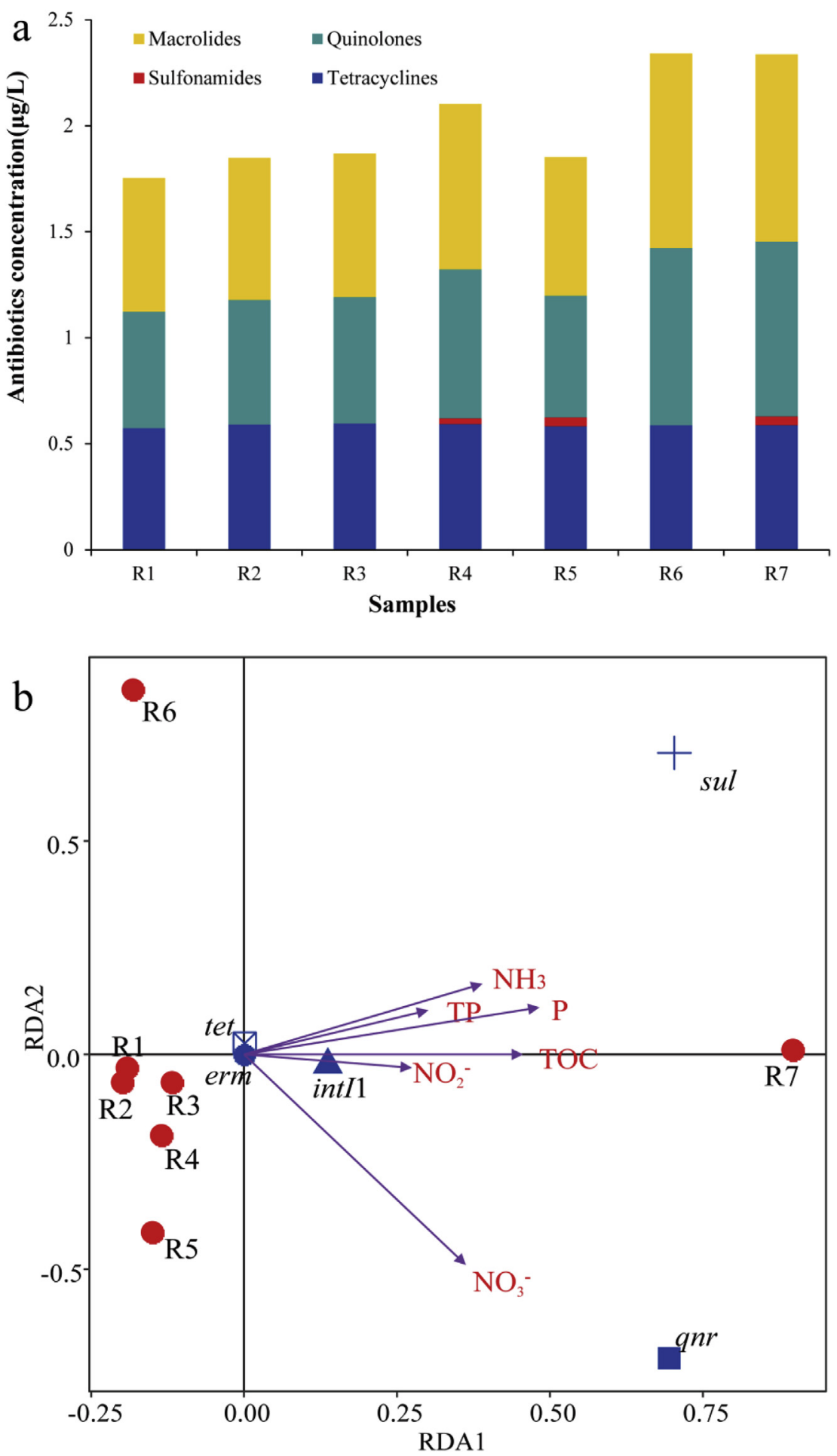

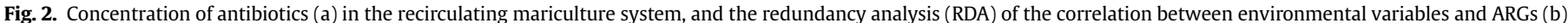

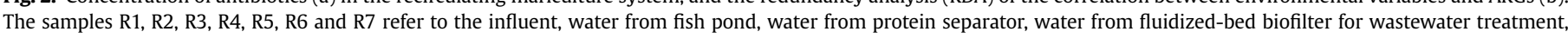
water from re-oxygenation pool, the recycled water, and feces and residual baits collected from the fish ponds and the protein separator, respectively.

and Zhang, 2013), types of ARGs (Wu et al., 2016; Shi et al., 2013), and operating parameters (Tian et al., 2016). The investigation on the removal of ARGs in different treatment units of four wastewater treatment plants showed that the biological treatment unit played the most important role in ARGs removal (1.2-1.8 orders of magnitude), and followed by UV disinfection while primary physical treatment units could hardly remove any ARGs (Wen et al.,
2016). The removal of ARGs using different disinfection processes including chlorination and UV disinfection had been reported (Wen et al., 2016; Zhuang et al., 2015). Zhuang et al. (2015) found that chlorination made $2.98-3.24 \mathrm{log}$ reduction, UV irradiation made 2.48-2.74 log reduction, ozonation made $1.68-2.55 \log$ reduction on sul1, tetG and intI1. In advanced treatment systems, 1-3 orders of magnitude of reductions in ARGs were observed in constructed 
Table 2

Pearson correlation analysis on antibiotics and ARGs.

\begin{tabular}{|c|c|c|c|c|c|c|c|c|c|}
\hline & sul & tet & $q n r$ & erm & intI1 & Sulfonamides & Tetracyclines & Quinolones & Macrolides \\
\hline sul & 1.000 & & & & & & & & \\
\hline tet & $0.919^{* *}$ & 1.000 & & & & & & & \\
\hline$q n r$ & $0.999^{* *}$ & $0.906^{* *}$ & 1.000 & & & & & & \\
\hline erm & $0.992^{* *}$ & $0.960 * *$ & $0.987^{* *}$ & 1.000 & & & & & \\
\hline intI1 & $0.997^{* *}$ & $0.923^{* *}$ & $0.997^{* *}$ & $0.992^{* *}$ & 1.000 & & & & \\
\hline Sulfonamides & -0.144 & -0.226 & -0.141 & -0.154 & -0.162 & 1.000 & & & \\
\hline Tetracyclines & -0.149 & -0.230 & -0.146 & -0.158 & -0.167 & $1.000^{* *}$ & 1.000 & & \\
\hline Quinolones & -0.123 & -0.194 & -0.122 & -0.129 & -0.142 & $0.999^{* *}$ & $0.999^{* *}$ & 1.000 & \\
\hline Macrolides & -0.129 & -0.201 & -0.127 & -0.135 & -0.147 & $0.999 * *$ & $0.999^{* *}$ & $1.000^{* *}$ & 1.000 \\
\hline
\end{tabular}

** Means the significant level at $\mathrm{p}<0.01$.

*Means the significant level at $\mathrm{p}<0.05$.

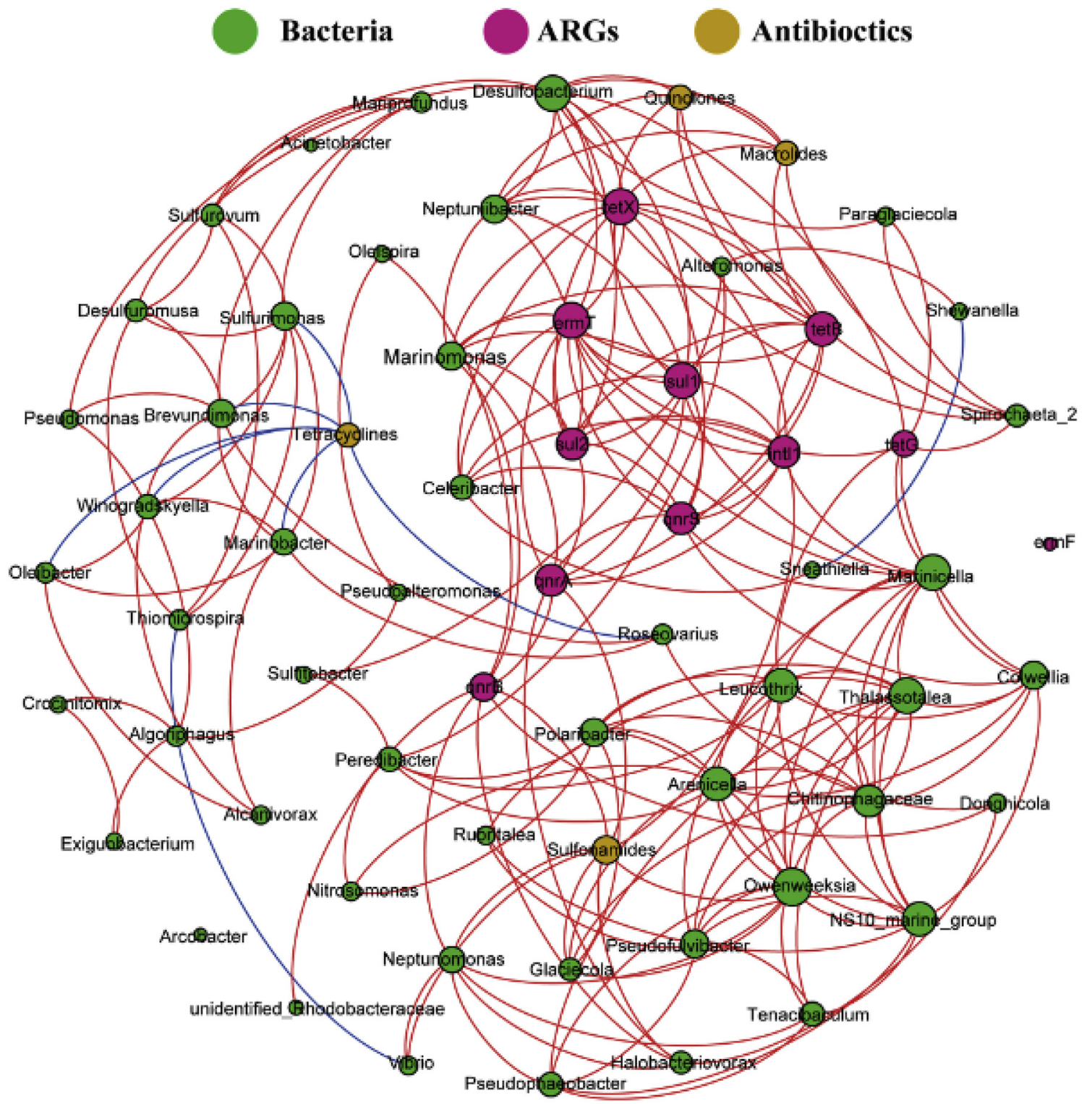

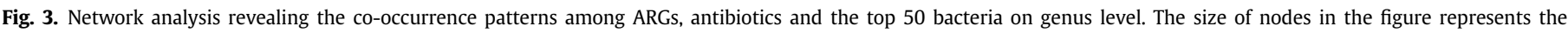

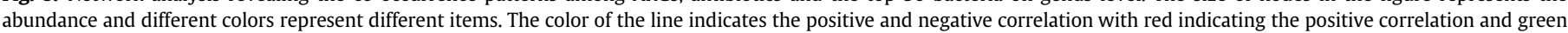

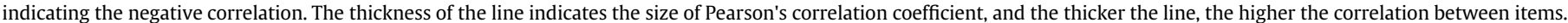
(For interpretation of the references to color in this figure legend, the reader is referred to the Web version of this article.)

wetlands, $0.6-1.2$ orders of magnitude of reductions in ARGs were observed in biological aerated filter (Chen and Zhang, 2013).

The intI1 gene in R7 sample possessed the highest abundance
(Fig. 4a), indicating that the discharge of feces and residual baits from the mariculture farm was an important source of intI 1 in the environment. The abundances of intI1 of samples R5 and R4 were 


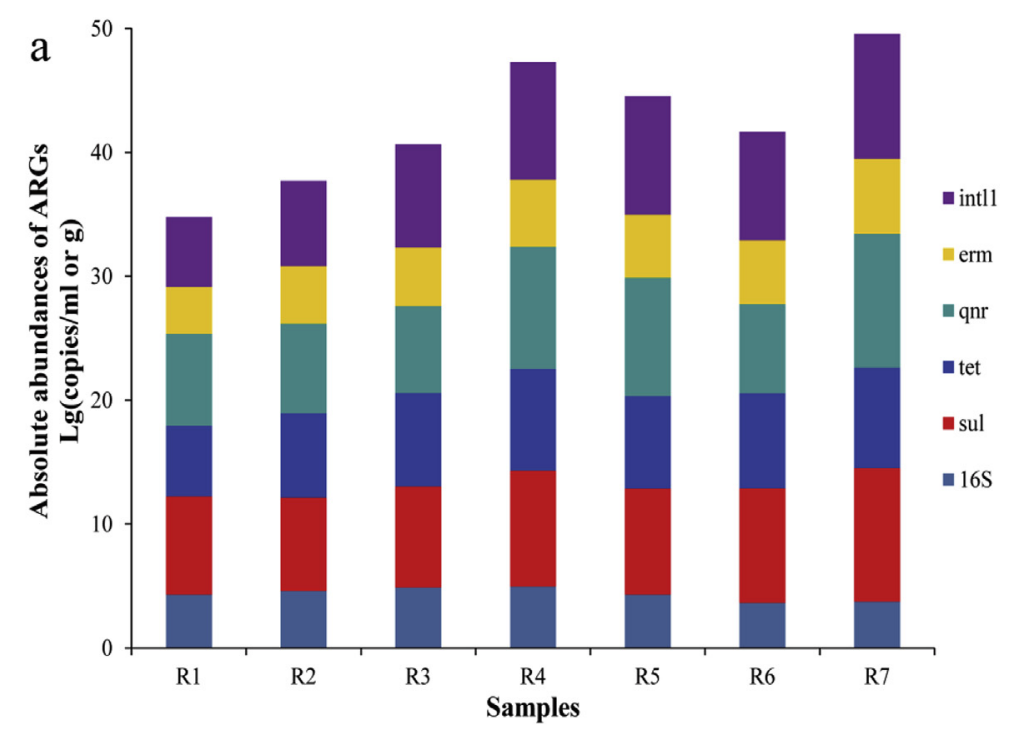

$\mathrm{b}$

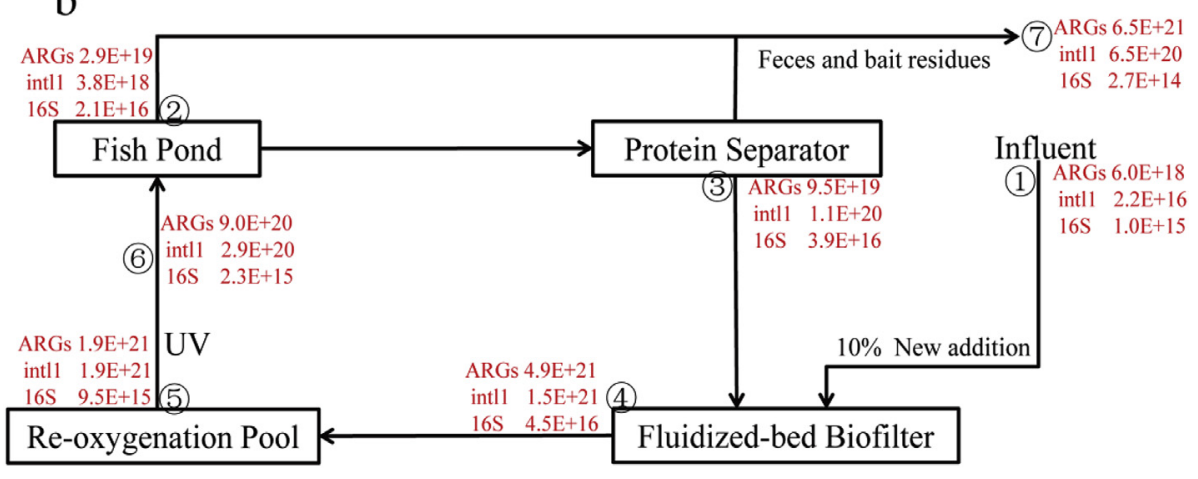

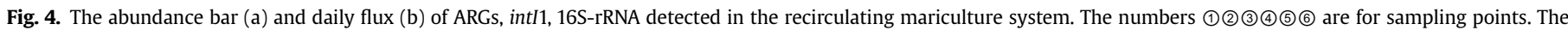

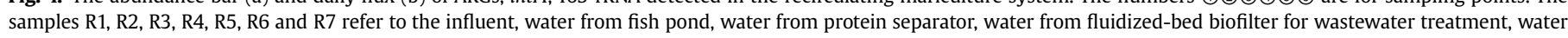
from re-oxygenation pool, the recycled water, and feces and residual baits collected from the fish ponds and the protein separator, respectively.

much higher than those of R1, R2, and R3, indicating that fluidizedbed biofilter and aerobic pool were important breeding ground for intI 1 that could not be eliminated by biological aeration. The abundance of intI 1 in R6 was lower than that in R5, suggesting that UV sterilization could eliminate intI 1 to some extent. The abundances of intI1 in samples R1 and R2 were much lower than those in other samples, which was the same to ARGs. The intI1 gene was detected $0.3-2.7$ orders of magnitude removal efficiency in four wastewater treatment plants (Wen et al., 2016). In addition, a twophase thermophilic digestion reduced the intI 1 with a $0.1-0.72 \log$ unit (Wu et al., 2016). The results suggested that: (1) the abundance of intI1 gene in environmental water was far lower than that in the mariculture system; (2) the mariculture system was an important source of intI1 gene; (3) fluidized-bed biofilter, aeration pool and protein separator were important breeding grounds for intI1 gene; (4) intI1 was not produced during the A. salmon breeding process; (5) int gene preferred to adhere to the feces and baits residue.

The analysis on the daily flux of ARGs and intI 1 genes through every unit of the recirculating system showed that fluidized-bed biofilter was the most important breeding ground for ARGs and intI1 (Fig. 4b). The highest ARGs flux $\left(6.5 \times 10^{21}\right.$ copies/d) through various units was observed in R7, indicating that the mariculture system emitted a large amount of ARGs into the environment per day through the discharge of feces and residual baits. Secondly, the daily flux of ARGs in R4 was much higher than that in R1 and R3, which confirmed that fluidized-bed biofilter was the most important breeding ground for ARGs. Compared with R4, the flux of ARGs in R6 deceased sharply to $9.0 \times 10^{20}$ copies/d, indicating that ARGs could be reduced efficiently by UV sterilization. The UV sterilization could remove the ARGs since it can eliminate some resistant bacteria (Macauley et al., 2006). The amount of ARGs in R2 was much lower than that in R6, showing that ARGs was significantly reduced. One rational explanation for this reduction was that a large amount of ARGs were absorbed in the feces and baits since the largest flux was observed in the discharge of feces and residual baits. Although the recirculating systems have been considered as a promising approach for preventing the coastal water pollution of antibiotics and ARGs, the highest abundance and flux of ARGs in the discharged faces and residual baits indicated that the recirculating mariculture system was also an important ARGs reservoir in coastal area.

\section{Conclusions}

Ten ARGs subtypes including sul (sul1, sul2), tet (tetB, tetG, tetX), $q n r(q n r \mathrm{~A}, q n r \mathrm{~B}, q n r \mathrm{~S})$, and erm (erm $\mathrm{F}$, erm $\mathrm{T})$ were detected in recirculating mariculture farm. The sul 1 and sul2 were the most abundant ARGs. No correlation existed between antibiotics and ARGs. Some bacteria were positively correlated with two or more ARGs. The fluidized-bed biofilters in the recirculating system were the main breeding ground for ARGs while the UV sterilization reduced ARGs. The discharge of the feces and residual baits with 
abundant ARGs was the main gateway for ARGs in the recirculating mariculture system to enter the environments.

\section{Acknowledgements}

This work was supported by National Natural Science Foundation of China (No. 41877131), One Hundred Talents Program of Chinese Academy of Sciences (Y629041021), Taishan Scholar Program of Shandong Province (No. tsqn201812116), and Two-Hundred Talents Plan of Yantai (Y739011021), Research Program of CAS Key Laboratory of Coastal Environmental Processes and Ecological Remediation (No. 1189010002). The authors would like to thank the reviewers for their valuable suggestions and comments on the manuscript.

\section{Appendix A. Supplementary data}

Supplementary data to this article can be found online at https://doi.org/10.1016/j.envpol.2019.02.062.

\section{References}

An, X., Chen, Q., Zhu, D., Su, J.Q., 2018. Distinct effects of struvite and biochar amendment on the class 1 integron antibiotic resistance gene cassettes in phyllosphere and rhizosphere. Sci. Total Environ. 631-632, 668-676. https:// doi.org/10.1016/j.scitotenv.2018.03.034.

Bennett, J.L., Mackie, A.L., Park, Y., Gagnon, G.A., 2018. Advanced oxidation processes for treatment of $17 \beta$-Estradiol and its metabolites in aquaculture wastewater Aquacult. Eng. 83, 40-46. https://doi.org/10.1016/j.aquaeng.2018.08.003.

Chen, H., Zhang, M., 2013. Effects of advanced treatment systems on the removal of antibiotic resistance genes in wastewater treatment plants from Hangzhou, China. Environ. Sci. Technol. 47, 8157-8163. https://doi.org/10.1021/es401091y.

Chen, Q., An, X., Li, H., Su, J., Ma, Y., Zhu, Y.G., 2016. Long-term field application of sewage sludge increase the abundance of antibiotic resistance genes in soil. Environ. Int. 92-93, 1-10. https://doi.org/10.1016/j.envint.2016.03.026.

Gao, P., Mao, D., Luo, Y., Wang, L., Xu, B., Xu, L., 2012a. Occurrence of sulfonamide and tetracycline-resistant bacteria and resistance genes in aquaculture environment. Water Res. 46, 2355-2364. https://doi.org/10.1016/j.watres.2012.02. 004.

Gao, P., Munir, M., Xagoraraki, I., 2012b. Correlation of tetracycline and sulfonamide antibiotics with corresponding resistance genes and resistant bacteria in a conventional municipal wastewater treatment plant. Sci. Total Environ. 421-422, 173-183. https://doi.org/10.1016/j.scitotenv.2012.01.061.

Gao, Q., Li, Y., Yue, Y., Min, M., Peng, S., Shi, Z., Gao, Y., 2018. Diverse and abundant antibiotic resistance genes from mariculture sites of China's coastline. Sci. Total Environ. 630, 117-125. https://doi.org/10.1016/j.scitotenv.2018.02.122.

Huang, Y., Cheng, M., Li, W., Wu, L., Chen, Y., Luo, Y., Christie, P., Zhang, H., 2013. Simultaneous extraction of four classes of antibiotics in soil, manure and sewage sludge and analysis by liquid chromatography-tandem mass spectrometry with the isotopelabelled internal standard method. Anal. Methods 5, 3721-3731. https://doi.org/10.1039/C3AY40220G.

Jang, H.M., Kim, Y.B., Choi, S., Lee, Y., Shin, S.G., Unno, T., Kim, Y.M., 2018. Prevalence of antibiotic resistance genes from effluent of coastal aquaculture, South Korea. Environ. Pollut. 233, 1049-1057. https://doi.org/10.1016/j.envpol.2017.10.006.

Jia, S., Zhang, X.X., Miao, Y., Zhao, Y., Ye, L., Li, B., Zhang, T., 2017. Fate of antibiotic resistance genes and their associations with bacterial community in livestock breeding wastewater and its receiving river water. Water Res. 124, 259-268. https://doi.org/10.1016/j.watres.2017.07.061.

Jiao, Y.N., Chen, H., Gao, R.X., Zhu, Y.G., Rensing, C., 2017. Organic compounds stimulate horizontal transfer of antibiotic resistance genes in mixed wastewater treatment systems. Chemosphere 184, 53-61. https://doi.org/10.1016/j. chemosphere.2017.05.149.

Lu, J., Wu, J., Zhang, C., Zhang, Y., Lin, Y., Luo, Y., 2018. Occurrence, distribution, and ecological-health risks of selected antibiotics in coastal waters along the coastline of China. Sci. Total Environ. 644, 1469-1476. https://doi.org/10.1016/j. scitotenv.2018.07.096.

Luo, Y., Mao, D.Q., Rysz, M., Zhou, D.X., Zhang, H.J., Xu, L., Alvarez, P.J.J., 2010. Trends in antibiotic resistance genes occurrence in the Haihe River, China. Environ. Sci. Technol. 44, 7220-7225. https://doi.org/10.1021/es100233w.

Macauley, J.J., Qiang, Z., Adams, C.D., Surampalli, R., Mormile, M.R., 2006. Disinfection of swine wastewater using chlorine, ultraviolet light and ozone. Water Res. 40, 2017-2026. https://doi.org/10.1016/j.watres.2006.03.021.

Mao, D., Yu, S., Rysz, M., Luo, Y., Yang, F., Li, F., Hou, J., Mu, Q., Alvarez, P.J.J., 2015. Prevalence and proliferation of antibiotic resistance genes in two municipal wastewater treatment plants. Water Res. 85, 458-466. https://doi.org/10.1016/j. watres.2015.09.010.

Molton, J.S., Tambyah, P.A., Ang, B.S.P., Ling, M.L., Fisher, D.A., 2013. The global spread of healthcare-associated multidrug-resistant bacteria: a perspective from Asia. Clin. Infect. Dis. 56, 1310-1318. https://doi.org/10.1093/cid/cit020.

Pei, R., Kim, S., Carlson, K.H., Pruden, A., 2006. Effect of river landscape on the sediment concentrations of antibiotics and corresponding antibiotic resistance genes (ARG). Water Res. 40, 2427-2435. https://doi.org/10.1016/j.watres.2006. 04.017.

Peng, S., Zhou, B., Wang, Y., Lin, X., Wang, H., Qiu, C., 2016. Bacteria play a more important role than nutrients in the accumulation of tetracycline resistance in manure-treated soil. Biol. Fertil. Soils 52, 655-663. https://doi.org/10.1007/ s00374-016-1105-9.

Piedrahita, R.H., 2003. Reducing the potential environmental impact of tank aquaculture efluents through intensification and recirculation. Aquaculture 226, 35-44. https://doi.org/10.1016/S0044-8486(03)00465-4.

Pruden, A., Pei, R., Storteboom, H., Carlson, K.H., 2006. Antibiotic resistance genes as emerging contaminants: studies in northern Colorado. Environ. Sci. Technol. 40, 7445-7450. https://doi.org/10.1021/es0604131.

Sapkota, A., Sapkota, A.R., Kucharski, M., Burke, J. McKenzie, S., Walker, P. Lawrence, R., 2008. Aquaculture practices and potential human health risks: current knowledge and future priorities. Environ. Int. 34, 1215-1226. https:// doi.org/10.1016/j.envint.2008.04.009.

Sharma, V.K., Johnson, N., Cizmas, L., McDonald, T.J., Kim, H., 2015. A review of the influence of treatment strategies on antibiotic resistant bacteria and antibiotic resistance genes. Chemosphere 150, 702-714. https://doi.org/10.1016/j. chemosphere.2015.12.084.

Shi, P., Jia, S., Zhang, X.X., Zhang, T., Cheng, S., Li, A., 2013. Metagenomic insights into chlorination effects on microbial antibiotic resistance in drinking water. Water Res. 47, 111-120. https://doi.org/10.1016/j.watres.2012.09.046.

Su, H., Liu, S., Hu, X., Xu, X., Xu, W., Xu, Y., Li, Z., Wen, G., Liu, Y., Cao, Y., 2017. Occurrence and temporal variation of antibiotic resistance genes (ARGs) in shrimp aquaculture: ARGs dissemination from farming source to reared organisms. Sci. Total Environ. 607-608, 357-366. https://doi.org/10.1016/j. scitotenv.2017.07.040.

Sui, Q., Zhang, J., Tong, J., Chen, M., Wei, Y., 2017. Seasonal variation and removal efficiency of antibiotic resistance genes during wastewater treatment of swine farms. Environ. Sci. Pollut. Res. 24, 9048-9057. https://doi.org/10.1007/s11356015-5891-7.

Tian, Z., zhang, Y., Yu, B., Yang, M., 2016. Changes of resistome, mobilome and potential hosts of antibiotic resistance genes during the transformation of anaerobic digestion from mesophilic to thermophilic. Water Res. 98, 261-269. https://doi.org/10.1016/j.watres.2016.04.031.

van Rijn, J., 2013. Waste treatment in recirculating aquaculture systems. Aquacult Eng. 53, 49-56. https://doi.org/10.1016/j.aquaeng.2012.11.010.

Wang, J.H., Lu, J., Zhang, Y.X., Wu, J., Luo, Y., Liu, H., 2018. Metagenomic analysis of antibiotic resistance genes in coastal industrial mariculture systems. Bioresour. Technol. 253, 235-243. https://doi.org/10.1016/j.biortech.2018.01.035.

Wen, Q., Yang, L., Duan, R., Chen, Z., 2016. Monitoring and evaluation of antibiotic resistance genes in four municipal wastewater treatment plants in Harbin, Northeast China. Environ. Pollut. 212, 34-40. https://doi.org/10.1016/j.envpol. 2016.01.043.

Wu, N., Qiao, M., Zhang, B., Cheng, W., Zhu, Y.G., 2010. Abundance and diversity of tetracycline resistance genes in soils adjacent to representative swine feedlots in China. Environ. Sci. Technol. 44, 6933-6939. https://doi.org/10.1021/ es1007802.

Wu, Y., Cui, E., Zuo, Y., Cheng, W., Rensing, C., Chen, H., 2016. Influence of two-phase anaerobic digestion on fate of selected antibiotic resistance genes and class 1 integrons in municipal wastewater sludge. Bioresour. Technol. 211, 414-421. https://doi.org/10.1016/j.biortech.2016.03.086.

Xiong, W., Sun, Y., Zhang, T., Ding, X., Li, Y., Wang, M., Zeng, Z., 2015. Antibiotics, antibiotic resistance genes, and bacterial community composition in fresh water aquaculture environment in China. Microb. Ecol. 70, 425-432. https:// doi.org/10.1007/s00248-015-0583-X.

Xu, J., Xu, Y., Wang, H., Guo, C., Qiu, H., He, Y., Zhang, Y., Li, X., Meng, W., 2015. Occurrence of antibiotics and antibiotic resistance genes in a sewage treatment plant and its effluent-receiving river. Chemosphere 119, 1379-1385. https://doi. org/10.1016/j.chemosphere.2014.02.040.

Xu, Y., Xu, J., Mao, D., Luo, Y., 2017a. Effect of the selective pressure of sub-lethal level of heavy metals on the fate and distribution of ARGs in the catchment scale. Environ. Pollut. 220, 900-908. https://doi.org/10.1016/j.envpol.2016.10. 074

Xu, Y.B., Hou, M.Y., Li, Y.F., Huang, L., Ruan, J.J., Zheng, L., Qiao, Q.X., Du, Q.P., 2017b. Distribution of tetracycline resistance genes and AmpC $\beta$-lactamase genes in representative non-urban sewage plants and correlations with treatment processes and heavy metals. Chemosphere 170, 274-281. https://doi.org/10.1016/j. chemosphere.2016.12.027.

Yang, L., Chou, L.-S., Shieh, W.K., 2001. Biofilter treatment of aquaculture water for reuse applications. Water Res. 35, 3097-3108. https://doi.org/10.1016/S00431354(01)00036-7.

Yang, Y., Xu, C., Cao, X., Lin, H., Wang, J., 2017. Antibiotic resistance genes in surface water of eutrophic urban lakes are related to heavy metals, antibiotics, lake morphology and anthropic impact. Ecotoxicology 26, 831-840. https://doi.org/ 10.1007/s10646-017-1814-3.

Zhang, Y., Zhuang, Y., Geng, J., Ren, H., Xu, K., Ding, L., 2016. Reduction of antibiotic resistance genes in municipal wastewater effluent by advanced oxidation processes. Sci. Total Environ. 550, 184-191. https://doi.org/10.1016/j.scitotenv. 2016.01.078.

Zheng, J., Zhou, Z., Wei, Y., Chen, T., Feng, W., Chen, H., 2018. High-throughput 
profiling of seasonal variations of antibiotic resistance gene transport in a periurban river. Environ. Int. 114, 87-94. https://doi.org/10.1016/j.envint.2018.02. 039.

Zhu, Y.G., Zhao, Y., Li, B., Huang, C.L., Zhang, S.Y., Yu, S., Chen, Y.Y., Zhang, T., 2017. Continental-scale pollution of estuaries with antibiotic resistance genes. Nat.
Microbiol. 2, 16270. https://doi.org/10.1038/nmicrobiol.2016.270.

Zhuang, Y., Ren, H., Geng, J., Zhang, Y., Zhang, Y., Ding, L., Xu, K., 2015. Inactivation of antibiotic resistance genes in municipal wastewater by chlorination, ultraviolet, and ozonation disinfection. Environ. Sci. Pollut. Res. 22, 7037-7044. https://doi org/10.1007/s11356-014-3919-z. 